\title{
Role of biomarkers for early detection of ovarian cancer recurrence
}

\author{
Pierluigi Giampaolino $^{1}$, Virginia Foreste ${ }^{2}$, Luigi Della Corte ${ }^{2}$, Claudia Di Filippo ${ }^{2}$, Giuseppe Iorio ${ }^{2}$, \\ Giuseppe Bifulco²
}

${ }^{1}$ Department of Public Health, School of Medicine, University of Naples Federico II, Naples, Italy; ${ }^{2}$ Department of Neuroscience, Reproductive Sciences and Dentistry, School of Medicine, University of Naples Federico II, Naples, Italy

Contributions: (I) Conception and design: P Giampaolino; (II) Administrative support: G Bifulco; (III) Provision of study materials: V Foreste; (IV) Collection and assembly of data: C Di Filippo, G Iorio; (V) Data analysis and interpretation: V Foreste, L Della Corte; (VI) Manuscript writing: All authors; (VII) Final approval of manuscript: All authors.

Correspondence to: Pierluigi Giampaolino, MD, PhD. Department of Public Health, School of Medicine, University of Naples Federico II, Naples, Italy. Email: pgiampaolino@gmail.com.

\begin{abstract}
Ovarian cancer is frequently diagnosed at an advanced stage and a fraction of these patients fail to respond to primary therapy and relapses in $70 \%$ of cases. On account of the high recurrence probability and the poor outcomes after recurrence, there is an urgent need to predict progression as early as possible and thus found the strategies to detect and prevent a recurrence. Considering that biomarkers have contributed to the management of ovarian cancer by distinguishing benign and malignant pelvic masses and monitoring response to treatment, in this review, we aim to discuss the latest evidence reported in the literature about the use of biomarkers to detect OC recurrence. In detail, we summarized all the evidence of the most quoted biomarkers like HE4, osteopontin, mesothelin (MSLN), Folate receptor $\alpha$ (FOLR1), paraneoplastic antigens, miRNA, cancer stem cells (CSCs) and a combination of them to evaluate their role as prognostic biomarkers for ovarian cancer recurrence.
\end{abstract}

Keywords: Biomarkers; ovarian cancer; recurrence; early detection

Submitted Mar 29, 2020. Accepted for publication Jun 12, 2020.

doi: $10.21037 / g s-20-544$

View this article at: http://dx.doi.org/10.21037/gs-20-544

\section{Introduction}

Ovarian cancer (OC) is the leading cause of gynecological cancer-associated death; indeed, according to reports from the National Cancer Institute (NCI), about 140,000 people die each year from OC worldwide (1). This disease is named the "silent killer" related to the fact that cancer becomes widespread without the occurrence of symptoms, and even if they are present (2), these symptoms are shared with a variety of more common benign gastrointestinal, genitourinary and gynecological conditions, making them difficult to attribute to ovarian cancer $(3,4)$. When the disease is detected in stage I (still limited to the ovaries), according to the International Federation of Obstetrics and Gynecology (FIGO) classification (5), up to $90 \%$ of patients can be cured successfully with currently available surgery and chemotherapy (6). Even when the disease has spread to the pelvis in stage II, $70 \%$ of patients can be cured, but when the disease has spread throughout the abdominal cavity or beyond can be cured in less than $20 \%$ of cases. Unfortunately, despite the improvement in overall survival for OC patients, a fraction of patients with advanced-stage disease fails to respond to primary therapy and relapses in $70 \%$ of cases (7-9).

On account of the high recurrence probability and the poor outcomes after recurrence, there is an urgent need to predict progression as early as possible and found strategies to early recognize and prevent recurrence (10). Transvaginal ultrasonography and cancer antigen 125 (CA-125) are the common methods for the detection of ovarian cancer, and 
biomarkers as CA125, Human epididymis protein 4 (HE4), apolipoprotein $\mathrm{A} 1$, transthyretin, transferrin, and $\beta 2$ macroglobulin have contributed to the Risk of Malignancy index (RMI), Risk of Malignancy Algorithm (ROMA), OVA1 algorithms and International Ovarian Tumor Analysis (IOTA) to distinguish benign from malignant disease (11). Over the decades, different biomarkers have come forward and the rising number of studies based on the combination of different biomarkers along with CA125 seems to be encouraging, but none of the biomarkers in clinical use for early detection of OC, including carcinoembryonic antigen (CEA), CA125, carbohydrate antigen 19-9 (CA19-9) and HE4 are effective due to a lack of sensitivity or specificity (12).

The sensitivity of a biomarker is measured by its ability to identify a patient with the disease correctly, as it will be present in diseased samples, and the specificity is measured by the ability to not be detected in healthy individuals (13). A biomarker with only one of these attributes will lead to false positives or false negatives, respectively (13). Current evidence has been devoted to discover the role of serum biomarkers in predict the risk of recurrence of ovarian cancer. In this way, biomarkers can be used not only as a diagnostic tool but also as a prognostic indicator that gives the estimate of disease recurrence and categorizes patients at different risk levels for specific outcomes (12). Nowadays, the clinical symptoms of relapse are determined by measuring the level of serum CA125, one of the most extensively used tumor biomarkers in standard clinical practice for disease surveillance (14). Levels of CA125 are correlated with response to treatment and it has been established that it can rise 4.8 months before clinical disease recurrence, providing time to pursue multiple courses of novel or conventional therapy (11), and a recent study showed, also a major benefit from early initiation of treatments (15). To make patients benefit from an early therapeutic intervention capable of prolonging the disease-free interval and improve overall survival, there is an urgent need of evidence sensitive biomarkers that can predict ovarian cancer recurrence with a sufficient lead time before the rise in CA125. Researches are evaluating several biomarkers like HE4, osteopontin (OPN), mesothelin (MSLN), Folate receptor $\alpha$ (FOLR1), paraneoplastic antigens, miRNA, cancer stem cells (CSCs) and a combination of them to evaluate their role as prognostic biomarkers for ovarian cancer recurrence. This narrative review has aimed to investigate the role of biomarkers for early detection of ovarian cancer recurrence, and to summarize the available evidence.

\section{Methods}

The data research was conducted using the following databases MEDLINE, EMBASE, Web of Sciences, Scopus, Clinical Trial. Gov, OVID and Cochrane Library querying for all articles related to ovarian cancer recurrence and ovarian biomarkers. The final literature search was performed in February 2020. Two authors (VF and CDF) independently screened titles and abstracts of studies obtained in the search. The studies were identified with the use of a combination of the following text words: "ovarian neoplasm" or "ovarian cancer" or "ovarian malignancy" or "secondary cytoreduction" or "recurrence" or "relapse" and "ovarian biomarkers". The selection criteria of this narrative review included randomized clinical trials, non-randomized controlled studies (observational prospective, retrospective cohort studies, case-control studies, case series) and review articles on the role of ovarian biomarkers in women with ovarian cancer recurrence.

\section{Prognostic biomarkers of recurrence}

Nowadays, non-invasive methods for early identification of OC recurrence are needed and there is growing interest in the evaluation of the role of serum biomarkers. To early identify relapses in a phase in which response rate to pharmacological treatments is higher (16), different biomarkers seem to be crucial for developing effective approaches to deal with ovarian cancer at the level of recurrence. The most quoted biomarkers are summarized below.

\section{CA125}

CA125 is a member of the mucin family glycoproteins, normally expressed in Mullerian and coelomic epithelial tissue derivatives, defined as a non-specific marker (17) considering that its levels can increase in various benign conditions, such as endometriosis, pelvic inflammatory disease, peritonitis, but also in malignant circumstances such as ovarian cancer (18). CA125 is one of the most extensively used biomarkers in standard clinical practice for epithelial OC (EOC) surveillance and to predict prognosis. Usually, a serum concentration of CA125 $>35 \mathrm{U} / \mathrm{mL}$ is suggestive of potential malignancies, with a $47 \%$ elevation in EOC early stage and an $80-90 \%$ elevations in advanced-stage (19), 
even if it can also be silent, as in about $20 \%$ of ovarian cancers (20). For CA125 as cancer surveillance, levels higher than $65 \mathrm{U} / \mathrm{mL}$ are associated with a lower 5 years' survival rate (21). The sensitivity and specificity of detecting early ovarian cancer recurrence using CA125 tumor marker alone with a threshold of $35 \mathrm{U} / \mathrm{mL}$ was $67.39 \%$ and $86.79 \%$ respectively (22).

CA125 appears to be suggestive when its pretreatment levels are compared with its post-treatment levels: a posttreatment decrease in CA125 values by half is usually associated with a favorable response to treatment while a doubling of values is indicative of drug resistance or disease progression in patients with CA125 levels that never normalize; in patients in whom CA125 values normalize after treatment, an increase above the threshold value of $35 \mathrm{U} / \mathrm{mL}$ can be considered suspect of progression or relapse (23).

However, a retrospective analysis conducted on 342 patients with ovarian cancer surgically treated, shows that among the patients who developed a recurrence, the median value of CA125 is $<35 \mathrm{U} / \mathrm{mL}(29.7 \mathrm{U} / \mathrm{mL})$. In particular, in three patients with CA125 values of $14.5,13.5$ and 20.9 U/mL, respectively, the PET examination found lesions located in the spleen, liver and pelvic region (15). Thus, a $10.5 \%$ increase in CA 125 levels may be predictive of disease progression and require a CT scan. Changes less than $0.5 \%$ are predictive of the absence of progression. If changes range from $0.5 \%$ to $10.5 \%$, and individualized clinical based approach is suggested (24). Increasing levels of CA125 precede the signs and symptoms of recurrence by $3-5$ months in as many as $70 \%$ of cases (25).

These findings agree with those of previous studies (26-28) confirming the fact that although having a good specificity in the diagnosis of recurrence, the CA125 does not have an optimal sensitivity since not all ovarian cancer produces elevated blood levels of CA125, particularly in mucinous cancer (29).

In contrast to these data, some authors believe that monitoring the CA125 levels has no clinical value for the follow up of recurrence for postoperative patients with epithelial OC (30). In particular, the RCT performed by Rustin et al. showed that early treatment of recurrent ovarian cancer based on rising CA125 did not improve overall survival when compared to treatment at clinical relapse. However, this study has several limitations: only chemotherapy was considered as early treatment and the impact of second-line cytoreductive surgery was not taken into consideration; changes of CA125 within the normal range were not considered, delaying detection of recurrence; in some cases, it been used a suboptimal therapy by current standards (31).

Indeed, both the European Society of Gynecologic Oncologists (ESGO) and the European Society of Medical Oncology (ESMO) advised against universally abandoning CA125 in the routine follow-up of all patients with ovarian cancer based on this single randomized trial because, in some patients, regular CA125 measurements may signal tumor growth before symptoms appear $(32,33)$. To date, CA125 is of a greater interest in a series of biomarkers panels, to obtain the best surveillance of ovarian cancer. According to recent evidence, the increase in CA125 levels during follow-up is associated with a specific localization of the recurrence, at the peritoneal and intra-abdominal level (lymph node, vaginal stump and cul de sac) (34). It seems that patients without the rise of CA125 level $(<35 \mathrm{U} / \mathrm{mL})$ had predominantly supradiaphragmatic lesions in the lung and brain. These findings suggest that during follow up for ovarian cancer greater emphasis should be given to computed tomography (CT) and magnetic resonance imaging (MRI), not only at the abdominal-pelvic level (34).

\section{HE4}

HE4 (35) is a protease inhibitor, primarily expressed in the reproductive and respiratory tracts but also characterized by good sensitivity and specificity in detecting OC, especially endometrioid (100\% overexpression) and serous subtypes (93\% overexpression) (36). It has been demonstrated that HE4 can regulate adhesion, migration, and growth of tumor cells through activation of the EGFR/MAPK signaling pathway (37). Up to now, HE4 has been approved by the Food and Drug Administration (FDA) to monitor disease progression or recurrence of EOC (38). What seems to be interesting, is that HE4 appears to be able to predict OC recurrence before CA125 in some patients and may be elevated in patients whose tumors fail to express CA125 (39) and Laskshmann et al. found that serum HE4 had equivalent sensitivity $(85.3 \%$ vs. $84.3 \%)$ but higher specificity $(91.4 \%$ vs. $70.2 \%)$ than serum CA125 in detecting recurrence and a lead time of 3 months over CA125 (40). Anastasi et al. (41) reported the increase in $\mathrm{HE} 4$ to precede the increase in CA- 125 from 5 to 8 months and coincide with the recurrence of the disease and Liao et al. (42) reported that HE4 values in urine became positive in advance of clinical recurrence in several women despite normality of serum levels of HE4 and CA-125. Recently, also a larger sample retrospective study confirmed that HE4 can 
detect a recurrence before CA125 (with a median of 126 days before clinical confirmation) and that while CA125 increased only in $50 \%$ of cases, $\mathrm{HE} 4$ increased in $75 \%$ of patients with recurrence (43). A combination of CA125 and HE4 could be more useful to monitor response to treatment and early detect relapses if compared to each marker used individually (44,45), indeed, by combining CA-125 and HE4, sensitivity is $76 \%$ with $100 \%$ specificity (46). In 2008, Havrilesky et al. have proposed panels including multiple biomarkers (HE4, MMP7, Glycodelin) to obtain better information in disease monitoring. The authors choose this panel selecting candidate biomarkers who met the following inclusion criteria: (I) over-expression of candidate gene in epithelial ovarian cancer relative to normal ovarian epithelium; (II) over-expression of the encoded protein in ovarian tissue; (III) localization of encoded proteins to extracellular compartment as membrane protein or secreted protein; (IV) discriminated ovarian cancer from normal sera utilizing prototype immunological assays. They found that this panel predicted disease recurrence before the elevation of CA125 in $56 \%$ of cases and in an equivalent time frame to CA125 in $41 \%$ of cases. In this way, the recurrence was identified from 6 to 69 weeks before the CA125 elevation (47). However, considering the lack of evaluation of the specificity of the biomarker panel, these results should be considered preliminary and subject to further evaluation. In 2012, another study suggests the use of HE4 in a panel; they select CA125, HE4, and CA72.4 together rather than one by one in the detection of disease relapse during follow-up (48), considering the evidence of the elevation of the serum tumor marker CA72.4 in EOC. The authors concluded that the combination of the individual biomarkers $\mathrm{HE} 4$ and CA72.4 performs better and reveals positivity in more than $75 \%$ of the patients at relapse in the follow-up period (48). The results listed so far are very promising, but more multicentric, randomized with large cohort studies are needed to validate and reinforce the role of $\mathrm{HE} 4$ in ovarian cancer recurrence.

\section{OPN}

OPN, a $44 \mathrm{kDa}$ calcium-binding glycoprotein extensively expressed in multiple cell-types and detectable in human body fluids, has emerged as an important potential biomarker for diagnosing and treating cancer. It has proven to be an important signaling agent in the development and progression of numerous malignant tumors, including breast, lung, gastric and melanoma, as well as ovarian cancer $(49,50)$.
Already in 2001, OPN was identified as a potential biomarker for OC by using a cDNA microarray system (51), even if its role in predicting the recurrence of ovarian cancer has not yet been sufficiently evaluated and only one older longitudinal study focused on this topic. In this study, 38 patients with ovarian cancer underwent post-treatment follow-up with the dosage of OPN and CA125. OPN levels decline after treatment, similar to CA125 levels. In the case of recurrence disease, OPN levels rose earlier compared to $\mathrm{CA} 125$, in $90 \%$ of cases: the OPN level began to rise 5 months, while CA125 did not increase until 6 months after surgical cytoreduction. The authors concluded that OPN may be a clinically useful adjunct to CA125 in detecting recurrent ovarian cancer, but additional clinical trials are needed to assess the potential clinical utility of adding OPN to routine CA125 measurement during followup of ovarian cancer (52).

\section{MSLN}

MSLN is a $40-\mathrm{kDa}$ glycoprotein, whose expression is limited to mesothelial cells present in the serous membranes of the pleural, pericardial, and peritoneal spaces (53). MSLN is hypothesized to be involved in cell adhesion and signaling (54) and, as shown by a recent study, it can promote the migrating and invasive capabilities of ovarian cancer cells by activating the ERK signal pathway (55).

Several studies have shown that MSLN is an independent prognostic factor for patients with ovarian carcinoma: the elevated levels of MSLN predict poorer overall survival (OS) also in patients with optimal debulking surgery $(56,57)$.

Although MSLN is an important prognostic factor, it has not yet proven to be a valid marker for assessing the recurrence risk of ovarian cancer. Shummer et al. (58) were the only ones that evaluate if MSLN is an acceptable remission marker. The study was conducted on 23 patients with ovarian cancer who underwent surgery and chemotherapy; the behavior of the markers (CA125, HE4, MMP7, and MSLN) was then assessed during remission and before the evaluation of recurrence. Of the 20 patients in whom a recurrence was observed, only 4 showed an increase in MSLN levels, but this elevation was also detected by CA125 and/or HE4 (58). This makes MSLN less suited as a marker for ovarian cancer monitoring.

\section{FOLR1}

FOLR1 is a glycophosphatidylinositol (GPI) anchored 
glycopolypeptide, which is in part released into the blood in a soluble form (soluble folate receptors, sFR) (59). A recent prospective study was the first to evaluate the role of this biomarker in ovarian cancer surveillance. The authors have monitored levelsof sFR and CA125 in 28 women with ovarian cancer who underwent optimal debulking. In 11 out of 28 patients it was observed that $\mathrm{sFR}$ was able to predict the recurrence as its levels never returned to the baseline or even increased, while CA125 levels remained low. Probably it can be indicative of a cluster of tumor cells characterized by high tumor FR expression and low CA125 (60).

However, other studies are needed to understand the real ability of this marker to predict the recurrence of ovarian cancer. Farran et al. (60) already have initiated a prospective clinical protocol to study sFR in both up-front diagnosis and surveillance of ovarian cancer.

\section{Paraneoplastic antigens}

A recent study showed the usefulness of paraneoplastic antigens as biomarkers for prediction of recurrence in ovarian cancer. Paraneoplastic antigens are expressed in both cells of the nervous system and tumor and can elicit a humoral immune response, with the consequent formation of onco-neural-antibodies. These antibodies could lead to the development of various neurological disorders called paraneoplastic syndromes (61). However, it can occur also in the absence of paraneoplastic symptoms leading to their diagnostic utility in asymptomatic subjects. Chatterjee et al. (14) have carried out a retrospective study in 21 ovarian cancer patients, to evaluate the sensitivity of a panel of 6 paraneoplastic antigens (HARS, CDR2, Ro52, 4B7, $4 \mathrm{H} 4$ and 5H6) to predict recurrence of ovarian cancer before the rise in CA125 level (cutoff $35 \mathrm{U} / \mathrm{mL}$ ) or to the radiologic indication of clinical recurrence. The result of this study showed that antibodies to the 4 antigens, HARS, Ro52, CDR2 and 5H6, predicted ovarian cancer recurrence with a sensitivity of $90.5 \%$ when CA125 levels were below the standard cutoff $(35 \mathrm{U} / \mathrm{mL})$. The average time for predicting recurrence obtained was 5.03 months before the clinical or symptomatic relapse (14). However, it must be emphasized that these markers have only been evaluated in a small number of patients in whom CA125 values are within the normal range.

\section{Future prospective: $m i R N A$ and CSCs}

Several miRNAs, small non-coding RNAs that downregulate the protein expression of target genes, have been detected in whole blood, plasma, serum and exosomes of patients with ovarian cancer. miRNA show differential expression across the different histological types of ovarian cancer and in the study by Prahm et al., a number of different miRNAs were shown to act as significant and independent predictors of predictive of overall survival (OS), time to progression (TTP), progression free survival (PFS), and chemotherapy resistance (62). The meta-analysis conducted by Shi et al. reveals that miR-200 family and miR-30 family could be promising prognostic biomarkers of ovarian cancer, considering that the expression level of miR-200 family showed significant association with OS (HR $=0.78,95 \%$ CI: 0.64-0.94) and higher expression of miR-30 family was associated with elevated OS/PFS for ovarian cancer (OS, HR $=0.43$, 95\% CI: $0.13-0.74$; PFS, HR $=0.76,95 \%$ CI: 0.64-0.87) (63). Upregulation of miR-205, miR-203, miR200b, miR-141 and miR-21 in endometrioid and serous subtypes, down-regulation of miR-145 in serous and clear cell carcinomas and miR-222 down-regulation in endometrioid and clear cell carcinomas were found (64). Recent studies have hypothesized the role of miRNAs as a prognostic marker for recurrent EOC after chemotherapy. The expression of hsa-miR-1273g-3p was found to be significantly down-regulated in recurrence EOC compare with healthy control groups (65). miR-200a, miR-200b, and miR-429 have significant potential as diagnostic markers in relapse (66). Therefore, identification of a series of mi-RNA as prognostic biomarkers may lead also to new screening tools. Although several reports demonstrate the suitability of circulating miRNAs as cancer biomarkers, these molecules are still considered insufficient for clinical applications, primarily due to the lack of large-scale validation and inconsistencies among detection devices (67).

Moreover, the biological characteristics and mechanisms of the different miRNAs in ovarian cancer may differ, and methods for accurately and absolutely quantifying miRNAs are not uniformly normal, which limited applicability of the pooled analysis. Therefore, a recent meta-analysis suggest that multiple miRNA panels can achieve better accuracy and the validation of these series of mi-RNA as prognostic biomarkers may lead also to new screening tools in the future (68).

Recent evidence in OC defines CSCs such as the main responsible for disease aggressiveness, drug resistance and tumor relapse. The existence of CSC was proposed a long time ago and evaluated in several studies (69). The concept states that tumor growth is powered by a small number 
Table 1 Main results of the review and implications for research

Main results

CA 125 is the most extensively used biomarkers in standard clinical practice for epithelial OC surveillance and to predict prognosis. To date, CA125 is of a greater interest in a series of biomarkers panels, to obtain the best surveillance of ovarian cancer

HE4 has been approved by the FDA to monitor disease progression or recurrence of EOC

A combination of CA125 and HE4 could be more useful to monitor response to treatment and early detect relapses if compared to each marker used individually

Limitations

The literature on this issue is very scant

Implications for future research

The efforts of researchers should be focused on:

Identification of reliable serum biomarkers for standardized surveillance strategies of recurrence

Additional clinical trials to assess the potential clinical utility of adding new biomarkers, such as OPN, MSLN and FOLR1, to routine CA125 measurement during follow up of ovarian cancer

Discover definitive markers for the identification of the different CSCs to develop new antigenic and molecular targets for therapy

OC, ovarian cancer; FDA, Food and Drug Administration; CA 125, Cancer Antigen 125; HE4, Human Epididymis Protein 4; OPN, osteopontin; MSLN, Mesothelin; FOLR1, Folate receptor alpha 1; CSCs, cancer stem cells.

of stem cells that seem to be involved in all phases of tumorigenesis: from initial development to metastasis, and therefore to the recurrence of the tumor (70). Regarding OC, ovarian CSCs have been isolated from OC cell lines, ascites, and primary and metastatic tumors (70). The reason to discuss CSCs in correlation to ovarian biomarkers is based on the fact that is possible to use antigenic and molecular targets of CSC as OC markers; in detail, it has been demonstrated that antigens like CD44, EpCAM and ALDH1 can be used as OC biomarkers of recurrence (12).

CD44 is a cell surface glycoprotein involved in cellcell interactions, adhesion and cell migration. CD44 has been well documented as a common CSC marker in many cancers and is involved in therapeutic resistance. It has been shown that CD44+ cells can differentiate and selfrenew with the characteristic of cancer cells (71), and that CD44+ and CD117+ cells are ovarian CSCs characterized by increased chemoresistance to taxane and platinum-based therapy (72).

ALDH enzymes belong to a family of enzymes involved in metabolic processes and are responsible for the oxidation of aldehydes in carboxylic acids (73). CSCs with high ALDH activity were shown to correlate with advanced tumor stage, grade and poor outcomes in OC patients $(74,75)$.

EpCAM (adhesion molecule of epithelial cells), is a transmembrane protein expressed essentially in human adenocarcinoma (76). EpCAM is significantly expressed in EOC tissues compared to normal ovarian tissues. Expression along with CD44 is associated with stage, grade, and metastasis of EOC. Metastatic and recurrent tumors expressed higher levels of EpCAM than primary ovarian carcinomas (77).

However, the biggest limit as biomarkers is that none of these current CSC markers are expressed exclusively by OC tissues, thus the necessity of combine in panel different markers. Rather, the use of markers expressed by CSCs in OC could be important to discover the mechanisms of chemoresistance, find therapeutic targets and develop new treatment modalities, especially in the case of metastasis or recurrence.

\section{Conclusions}

Notwithstanding the high recurrence rate of OC, no surveillance strategies of recurrence are still well defined and standardized (11). Up to date, many studies try to define the way to anticipate the diagnosis of OC recurrence with the objective of translate this results in survival and quality of life improvement, and it has been evaluated that biomarkers may play a key role in this scenario (12).

Currently, biomarkers such as CA125 have been used to monitor response to treatment and to detect recurrence, but even if it appears to be highly specific, it is not optimally sensitive for monitoring a complete response to primary therapy and can detect disease recurrence only 4.8 months before signs and symptoms develop clinically (25). Moving forward, since less time of the revelation of recurrence should be obtained, a growing interest in the role of new serum biomarkers has been observed, as for HE4 (44). Indeed, up to date, FDA has recently approved only the use of $\mathrm{HE} 4$ in OC follow-up together with CA-125, even if few studies are available to date about its use in this setting (Table 1).

The upcoming goal will be to found the most potent combination of biomarkers for screening that can detect recurrence as earlier as possible with high sensitivity and 
specificity, considering that the early treatment of disease recurrence seems to be the optimal way for improving survival. In the future, the definition of new biomarkers in addition to the use of new generation sequencing technology for malignant OC could also help to identify specific markers for molecular targets therapy, as OC stem cell-associated biomarkers suggest.

\section{Acknowledgments}

Funding: None.

\section{Footnote}

Provenance and Peer Review: This article was commissioned by the Guest Editor (Stefano Cianci) for the series "Ovarian Cancer Recurrence" published in Gland Surgery. The article was sent for external peer review organized by the Guest Editor and the editorial office.

Conflicts of Interest: All authors have completed the ICMJE uniform disclosure form (available at http://dx.doi. org/10.21037/gs-20-544). The series "Ovarian Cancer Recurrence" was commissioned by the editorial office without any funding or sponsorship. The authors have no other conflicts of interest to declare.

Ethical Statement: The authors are accountable for all aspects of the work in ensuring that questions related to the accuracy or integrity of any part of the work are appropriately investigated and resolved.

Open Access Statement: This is an Open Access article distributed in accordance with the Creative Commons Attribution-NonCommercial-NoDerivs 4.0 International License (CC BY-NC-ND 4.0), which permits the noncommercial replication and distribution of the article with the strict proviso that no changes or edits are made and the original work is properly cited (including links to both the formal publication through the relevant DOI and the license). See: https://creativecommons.org/licenses/by-nc-nd/4.0/.

\section{References}

1. Torre LA, Trabert B, DeSantis CE, et al. Ovarian cancer statistics, 2018. CA Cancer J Clin 2018;68:284-96.

2. Goff BA, Mandel L, Muntz HG, et al. Ovarian carcinoma diagnosis. Cancer 2000;89:2068-75.
3. Guadagno E, Pignatiello S, Borrelli G, et al. Ovarian borderline tumors, a subtype of neoplasm with controversial behavior. Role of $\mathrm{Ki} 67$ as a prognostic factor. Pathol Res Pract 2019;215:152633.

4. Giampaolino P, Della Corte L, Foreste V, et al. Unraveling a difficult diagnosis: the tricks for early recognition of ovarian cancer. Minerva Med 2019;110:279-91.

5. Prat J; FIGO Committee on Gynecologic Oncology. Staging classification for cancer of the ovary, fallopian tube, and peritoneum. Int J Gynaecol Obstet 2014;124:1-5.

6. Gueli Alletti S, Capozzi VA, Rosati A, et al. Laparoscopy vs. laparotomy for advanced ovarian cancer: a systematic review of the literature. Minerva Med 2019;110:341-57.

7. Gallotta V, Conte C, Giudice MT, et al. Secondary Laparoscopic Cytoreduction in Recurrent Ovarian Cancer: A Large, Single-Institution Experience. J Minim Invasive Gynecol 2018;25:644-650.

8. Cianci S, Ronsini C, Vizzielli G, et al. Cytoreductive surgery followed by HIPEC repetition for secondary ovarian cancer recurrence. Updates Surg 2019;71:389-394.

9. Chiofalo B, Bruni S, Certelli C, et al. Primary Debulking Surgery vs. Interval Debulking Surgery for Advanced Ovarian Cancer: Review of the Literature and MetaAnalysis; Minerva Med 2019;110:330-40.

10. Zhang $\mathrm{F}$, Zhang $\mathrm{Y}, \mathrm{Ke} \mathrm{C}$, et al. Predicting ovarian cancer recurrence by plasma metabolic profiles before and after surgery. Metabolomics 2018;14:65.

11. Yang WL, Lu Z, Bast RC Jr. The role of biomarkers in the management of epithelial ovarian cancer. Expert Rev Mol Diagn 2017;17:577-91.

12. Muinao T, Deka Boruah HP, Pal M. Diagnostic and Prognostic Biomarkers in ovarian cancer and the potential roles of cancer stem cells - An updated review. Exp Cell Res 2018;362:1-10.

13. Sölétormos G, Duffy MJ, Hassan SOA, et al. Clinical use of cancer biomarkers in epithelial ovarian cancer: updated guidelines from the European group on tumor markers (EGTM). Int J Gynecol Cancer 2016;26:43-51.

14. Chatterjee M, Hurley LC, Levin NK et al. Utility of paraneoplastic antigens as biomarkers for surveillance and prediction of recurrence in ovarian cancer. Cancer Biomark 2017;20:369-387.

15. Guo N, Peng Z. Does serum CA125 have clinical value for follow-up monitoring of postoperative patients with epithelial ovarian cancer? Results of a 12-year study. J Ovarian Res 2017;10:14.

16. Hiom SC. Diagnosing cancer earlier: reviewing the evidence for improving can- cer survival. Br J Cancer 
2015;112:S1-5.

17. Duraisamy S, Ramasamy S, Kharbanda S, et al. Distinct evolution of the human carcinoma-associated transmembrane mucins, MUC1, MUC4 AND MUC16. Gene 2006;373:28-34.

18. Sundar S, Neal RD, Kehoe SJ. Diagnosis of ovarian cancer. BMJ 2015;351:h4443.

19. Dupont, Tanwar MK, Thaler HT, et al. Early detection and prognosis of ovarian cancer using serum YKL-40. J Clin Oncol 2004;22:3330-9.

20. Buamah P. Benign conditions associated with raised serum CA-125 concentration. J Surg Oncol 2000;75:264-5.

21. Colaković S, Lukiç V, Mitroviç L, et al. Prognostic value of CA125 kinetics and half-life in advanced ovarian cancer. Int J Biol Markers 2000;15:147-52.

22. Yang ZJ, Zhao BB, Li L. The significance of the change pattern of serum CA125 level for judging prognosis and diagnosing recurrences of epithelial ovarian cancer. J Ovarian Res 2016;9:57.

23. Rustin GJ, Vergote I, Eisenhauer E, et al. Definitions for response and progression in ovarian cancer clinical trials incorporating RECIST 1.1 and CA 125 agreed by the Gynecological Cancer Intergroup (GCIG). Int J Gynecol Cancer 2011;21:419-23.

24. Giuliani M, Gui B, Valentini AL, et al. Early detection of recurrence or progression disease in patients with ovarian cancer after primary debulking surgery. Correlation between CT findings and CA 125 levels. Minerva Ginecol 2017;69:538-47.

25. Rustin GJ, Nelstrop AE, Tuxen MK, et al. Defining progression of ovarian carcinoma during follow-up according to CA 125: a North Thames Ovary Group Study. Ann Oncol 1996;7:361-4.

26. Low RN, Duggan B, Barone RM, et al. Treated ovarian cancer: MR imaging, laparotomy reassessment, and serum CA-125 values compared with clinical outcome at 1 year. Radiology 2005;235:918-26.

27. García-Velloso MJ, Jurado M, Ceamanos C, et al. Diagnostic accuracy of FDG PET in the follow-up of platinum-sensitive epithelial ovarian carcinoma. Eur J Nucl Med Mol Imaging 2007;34:1396-405.

28. Crawford SM, Peace J. Does the nadir CA125 concentration predict a longterm outcome after chemotherapy for carcinoma of the ovary? Ann Oncol 2005;16:47-50.

29. Scholler N, Urban N. CA125 in Ovarian Cancer. Biomark Med 2007;1:513-23.

30. Rustin GJ, van der Burg ME, Griffin CL, et al. Early versus delayed treatment of relapsed ovarian cancer (MRC OV05/EORTC 55955): a randomised trial. Lancet 2010;376:1155-63

31. Bast RC Jr. CA 125 and the detection of recurrent ovarian cancer: a reasonably accurate biomarker for a difficult disease. Cancer 2010;116:2850-3.

32. Verheijen RH, Cibula D, Zola P, et al. Cancer Antigen 125: Lost to Follow-Up? : A European Society of Gynaecological Oncology Consensus Statement. Int J Gynecol Cancer 2012;22:170-4.

33. Pignata S, Cannella L, Leopardo D, et al. Follow-up with CA125 after primary therapy of advanced ovarian cancer: in favor of continuing to prescribe CA125 during followup. Ann Oncol 2011;22 Suppl 8:140-4.

34. Wang PH, Huang YT, Ng KK, et al. Detecting recurrent ovarian cancer: revisit the values of whole-body CT and serum CA 125 levels. Acta Radiol 2019;60:1360-6.

35. Jing J, Gao Y. Urine biomarkers in the early stages of diseases: current status and perspective. Discov Med 2018;25:57-65.

36. Grayson K, Gregory E, Khan G, et al. Urine Biomarkers for the Early Detection of Ovarian Cancer - Are We There Yet? Biomark Cancer 2019;11:1179299X19830977.

37. Lu R, Sun X, Xiao R, et al. Human epididymis protein 4 (HE4) plays a key role in ovarian cancer cell adhesion and motility. Biochem Biophys Res Commun 2012;419:274-80.

38. Azzam AZ, Hashad DI, Kamel NA. Evaluation of HE4 as an extrabiomarker to CA125 to improve detection of ovarian carcinoma: is it time for a step forward?. Arch Gynecol Obstet 2013;288:167-72.

39. Plotti F, Guzzo F, Schirò T, et al. Role of human epididymis protein 4 (HE4) in detecting recurrence in CA125 negative ovarian cancer patients. Int J Gynecol Cancer 2019. [Epub ahead of print].

40. Lakshmanan M, Kumar V, Chaturvedi A et al. Role of serum HE4 as a prognostic marker in carcinoma of the ovary. Indian J Cancer 2019;56:216-21.

41. Anastasi E, Marchei GG, Viggiani V et al. HE4: a new potential early biomarker for the recurrence of ovarian cancer. Tumour Biol 2010;31:113-9.

42. Liao JB, Yip YY, Swisher EM, et al. Detection of the HE4 protein in urine as a biomarker for ovarian neoplasms: Clinical correlates. Gynecol Oncol 2015;137:430-5.

43. Abbink K, Zusterzeel PL, Geurts-Moespot AJ, et al. HE4 is superior to CA125 in the detection of recurrent disease in high-risk endometrial cancer patients. Tumour Biol 2018;40:1010428318757103.

44. Capriglione S, Luvero D, Piotti F, et al. Ovarian cancer 
recurrence and early detection: may $\mathrm{HE} 4$ play a key role in this open challenge? A systematic review of literature. Med Oncol 2017;34:164.

45. Wang Q, Wu Y, Zhang H, et al. Clinical Value of Serum HE4, CA125, CA72-4, and ROMA Index for Diagnosis of Ovarian Cancer and Prediction of Postoperative Recurrence. Clin Lab 2019;65.

46. Plotti F, Capriglione S, Terranova C, et al. Does HE4 have a role as biomarker in the recurrence of ovarian cancer? Tumour Biol 2012;33:2117-23.

47. Havrilesky LJ, Whitehead CM, Rubatt JM, et al. Evaluation of biomarker panels for early stage ovarian cancer detection and monitoring for disease recurrence. Gynecol Oncol 2008;110:374-82.

48. Granato T, Midulla C, Longo F, et al. Role of HE4, CA72.4, and CA125 in monitoring ovarian cancer. Tumour Biol 2012;33:1335-9.

49. Hu ZD, Wei TT, Yang M, et al. Diagnostic value of osteopontin in ovarian cancer: a meta-analysis and systematic review. PLoS One 2015;10:e0126444.

50. Zhao H, Chen Q, Alam A et al. The role of osteopontin in the progression of solid organ tumour. Cell Death Dis 2018;9:356-70.

51. Wong KK, Cheng RS, Mok SC, et al. Identification of differentially expressed genes from ovarian cancer cells by MICROMAX cDNA microarray system. Biotechniques 2001;30:670-5.

52. Schorge JO, Drake RD, Lee H, et al. Osteopontin as an adjunct to CA125 in detecting recurrent ovarian cancer. Clin Cancer Res 2004;10:3474-8.

53. Bast RC Jr, Klug TL, St John E, et al. A radioimmunoassay using a monoclonal antibody to monitor the course of epithelial ovarian cancer. N Engl J Med 1983;309:883-7.

54. Chang K, Pastan I. Molecular cloning of mesothelin, a differentiation antigen presents on mesothelium, mesotheliomas, and ovarian cancers. Proc Natl Acad Sci U S A 1996;93:136-40.

55. Chang MC, Chen CA, Chen PJ, et al. Mesothelin enhances invasion of ovarian cancer by inducing MMP7 through MAPK/ERK and JNK pathways. Biochem J 2012;442:293-302.

56. Cheng WF, Huang CY, Chang MC, et al. High mesothelin correlates with chemoresistance and poor survival in epithelial ovarian carcinoma. Br J Cancer 2009; 100:1144-53.

57. Cheng WF, Hung CF, Chai CY, et al. Generation and characterization of an ascitogenicmesothelin-expressing tumor model. Cancer 2007;110:420-31.
58. Schummer M, Drescher C, Forrest R, et al. Evaluation of ovarian cancer remission markers $\mathrm{HE} 4, \mathrm{MMP} 7$ and Mesothelin by comparison to the established marker CA125. Gynecol Oncol 2012;125:65-9.

59. Antony AC. Folate receptors. Annu Rev Nutr 1996;16:501-21.

60. Farran B, Albayrak S, Abrams J, et al. Serum folate receptor $\alpha(\mathrm{sFR})$ in ovarian cancer diagnosis and surveillance. Cancer Med 2019;8:920-7.

61. Chatterjee M, Hurley LC, Tainsky MA. Paraneoplastic antigens as biomarkers for early diagnosis of ovarian cancer. Gynecol Oncol Rep 2017;21:37-44.

62. Prahm KP, Høgdall C, Aarenstrup Karlsen M et al. Identification and validation of potential prognostic and predictive miRNAs of epithelial ovarian cancer. PLoS One 2018; 13:e0207319.

63. Shi M, Mu Y, Zhang H, et al. MicroRNA-200 and microRNA-30 family as prognostic molecular signatures in ovarian cancer: A meta-analysis. Medicine (Baltimore) 2018;97:e11505.

64. Iorio MV, Visone R, Di Leva G, et al. MicroRNA sig- natures in human ovarian cancer, Cancer Res 2007;67:8699-707.

65. Günel T, Gumusoglu E, Dogan B, et al. Potential biomarker of circulating hsa-miR-1273g-3p level for detection of recurrent epithelial ovarian cancer. Arch Gynecol Obstet 2018;298:1173-80.

66. Hu X, Macdonald DM, Huettner PC, et al. A miR-200 microRNA cluster as prognostic marker in advanced ovarian cancer, Gynecol Oncol 2009;114:457-64.

67. Yokoi A, Matsuzaki J, Yamamoto Y, et al. Integrated extracellular microRNA profiling for ovarian cancer screening; Nat Commun 2018;9:4319.

68. Wang X, Kong D, Wang C, et al. Circulating microRNAs as novel potential diagnostic biomarkers for ovarian cancer: a systematic review and updated meta-analysis. J Ovarian Res 2019;12:24.

69. Laganà AS, Colonese F, Colonese E, et al. Cytogenetic Analysis of Epithelial Ovarian Cancer's Stem Cells: An Overview on New Diagnostic and Therapeutic Perspectives; Eur J Gynaecol Oncol 2015;36:495-505.

70. Trott KR. Tumour stem cells: the biological concept and its application in cancer treatment. Radiother Oncol 1994;30:1-5.

71. Alvero AB, Chen R, Fu HH, et al. Molecular phenotyping of human ovarian cancer stem cells unravels the mechanisms for repair and chemoresistance. Cell Cycle 2009;8:158-66. 
72. Zhang S, Balch C, Chan MW, et al. Identification and characterization of ovarian cancer-initiating cells from primary human tumors. Cancer Res 2008;68:4311-20.

73. Marcato P, Dean CA, Giacomantonio CA, et al. Aldehyde dehydrogenase: its role as a cancer stem cell marker comes down to the specific isoform. Cell Cycle 2011;10:1378-84.

74. Landen CN, Goodman B, Katre AA, et al. Targeting aldehyde dehydrogenase cancer stem cells in ovarian cancer. Mol Cancer Ther 2010;9:3186-99.

75. Sun Y, Jia X, Wu X. High Expressions of Lgr5 and ALDH1 in Primary Epithelial Ovarian Cancer Correlate with Advanced Tumor Stage and Grade as well as

Cite this article as: Giampaolino P, Foreste V, Della Corte L, Di Filippo C, Iorio G, Bifulco G. Role of biomarkers for early detection of ovarian cancer recurrence. Gland Surg 2020;9(3):11021111. doi: $10.21037 /$ gs-20-544
Poor Prognosis of the Patients. Gynecol Obstet Invest 2015;81:162-8.

76. Munz M, Baeuerle PA and Gires O. The emerging role of EpCAM in cancer and stem cell signaling. Cancer Res 2009;69:5627-9.

77. Bellone S, Siegel ER, Cocco E et al. Overexpression of epithelial cell adhesion molecule in primary, metastatic, and recurrent/chemotherapy-resistant epithelial ovarian cancer: implications for epithelial cell adhesion molecule-specific immunotherapy. Int J Gynecol Cancer 2009;19:860-6. 